\title{
Sub-10 nm Crystalline Silicon Nanostructures by Electron Beam Induced Deposition Lithography
}

\author{
I. Sychugov, Y. Nakayama and K. Mitsuishi \\ Quantum Dot Research Center, National Institute for Materials Science, Sakura 3-13,
} Tsukuba, Ibaraki, 305-0003 Japan

\begin{abstract}
.
A novel top-down approach for the controllable fabrication of semiconductor nanostructures exhibiting quantum effects is described. By decomposing metal-rich precursor gas molecules with an electron beam a sub-10 $\mathrm{nm}$ metal pattern can be formed and subsequently transferred to a semiconductor substrate. In such a way monocrystalline silicon nanodots and nanowires are produced as revealed by the transmission electron microscopy. It is also shown how through controlled thermal or chemical oxidation the nanostructure surface can be passivated. By providing direct access to the sub-10 $\mathrm{nm}$ size range this method possesses promising potential for the application in quantum dot and nanoelectronics fields.
\end{abstract}

Keywords silicon nanostructures, quantum dot, nanowire, EBID PACS codes 81.07.Ta, 61.46.Df 


\section{Introduction}

Semiconductor properties change dramatically when the material characteristic size scales down to the nanometer range. When the size of a semiconductor entity becomes comparable to the exciton Bohr diameter (typically on the order of several nanometers) various quantum effects, such as a quantization of energy levels, begin to manifest [1]. Silicon is an industrial material of choice and its present manufacturing technology is steadily approaching the aforementioned size range [2]. The discreteness of energy levels in Si nanostructures was experimentally observed in nanodots by photoluminescence measurements with the detection of a narrow linewidth [3], and in nanowires by conductivity measurements revealing quantized conductance [4]. The change of material properties caused by quantum effects may impose insurmountable technological problems but may, just as well, provide new pathways for future applications unavailable with the bulk material. For example, a high sensitivity of silicon nanostructures to additional charges allows the operation of nanodots [5] and nanowires [6] as a charge storage media through the Coulomb blockade mechanism. At the same time, external charges adsorbed on the nanowire surface can remarkably alter its conductance, forming a foundation for the sensitive biomolecule detection [7]. It was also shown how unique properties of Si nanodots can be utilized in photovoltaics, surpassing inherent efficiency limits of the bulk material [8]. Light-emitting properties of silicon nanodots, on the other hand, can be used in toxic-free 
biolabeling [9]. Recently the potential for application of Si nanowires in the thermoelectric generation field was reported [10]. Again a significant improvement of the figure of merit, about two orders of magnitude compared to the bulk, was measured for the nanostructured material.

A controllable fabrication of silicon nanostructures exhibiting quantum effects, however, remains a difficult task. The optical diffraction limit restricts the use of optical lithography to the range of hundreds of nanometers. For certain applications, however, a combination with additional processing such as a selective anisotropic etching and a spacer technology can push the limit to tens of nanometers [11]. A conventional electron beam (e-beam) lithography patterning with a polymer resist, on the other hand, is typically reproducible in the range above $\sim$ 15-20 nm [12]. Hence, nanostructures fabricated by these methods require additional size-reduction steps to reach a sub-10 nm range [13]. A scanning probe lithography for local oxidation of silicon has similar limitations [14].

Electron beam induced deposition (EBID) is a nanofabrication technique capable of overcoming spatial resolution of the conventional e-beam lithography. Amorphous or polycrystalline nanostructures featuring dimensions less than $10 \mathrm{~nm}$ can be routinely formed from a number of precursor gases. High content metal nanostructures can be directly "written" 
on the sample surface by the electron beam and, hence, no additional metal deposition and lift-off steps are necessary for the fabrication of a metallic template [15]. Therefore this technique is drawing attention in applications such as circuitry, lithography and stencil mask repair as described in late reviews [16]. Recently it was shown how an EBID formed metal pattern can be transferred to the silicon substrate by ion milling [17] or dry etching [18], producing high aspect ratio Si nanostructures. It should be noted, though, that the ion beam etching [19] acting through physical sputtering introduces a lot of point damage or even amorphization to the substrate material. In this respect chemical sputtering, used in e.g. plasma etching, is much more preferable for the crystalline nanostructure fabrication.

Here we show an example of the extreme nanofabrication providing direct access to the sub-10 nm size range. Silicon nanodots and nanowires were produced from a thin silicon-on-insulator (SOI) substrate by EBID patterning and subsequent plasma etching. On top of direct access to the sub-10 $\mathrm{nm}$ size range this method provides additional flexibility in nanofabrication. With this technique various metal nanostructures can be formed in direct vicinity to their semiconductor counterparts for electric nanocontacts as well as for basic studies in plasmonics. In addition, only a short treatment is needed for surface passivation of nanostructures, allowing for better nanofabrication control. 


\section{Experimental methods}

A scanning electron microscope (SEM, JEOL JSM-6700-F) with a precursor powder evaporation unit was employed for EBID. A $30 \mathrm{keV}$ energy e-beam with $30 \mathrm{pA}$ sample current $\left(10 \mu \mathrm{A}\right.$ emission current) was used with $\mathrm{W}(\mathrm{CO})_{6}$ precursor gas at pressure $4 \cdot 10^{-4} \mathrm{~Pa}$ for all depositions. Silicon etching was done by $\mathrm{SF}_{6}$ reactive gas in the ULVAC CE-3001 plasma etching unit (gas flow $5 \mathrm{sccm}$, pressure $0.3 \mathrm{~Pa}$, discharge power $25 \mathrm{~W}$, bias power $13 \mathrm{~W}$ ). These conditions roughly correspond to $20 \mathrm{~nm} / \mathrm{min}$ etching rate. The metal mask was selectively removed by wet etching in a heated "aqua regia" solution $\left(3 \mathrm{HCl}: \mathrm{HNO}_{3}\right.$ at $\left.50^{\circ} \mathrm{C}\right)$. Thermal oxidation was carried out in a rapid thermal annealing machine (ULVAC QHC-P410) in pure oxygen atmosphere at $900{ }^{\circ} \mathrm{C}$ for 10 seconds. Nanostructures were visualized in a scanning transmission electron microscope (STEM, JEOL JEM-2500SES) equipped with electron energy loss spectroscopy (EELS) for elemental analysis. Samples for TEM observation were prepared using conventional focused ion beam milling (FIB) technique with a carbon protection layer deposition before the processing. Although the FIB itself can be used for nanopatterning by deposition [16] and etching [20], due to possible beam damage here it was used only for the TEM sample preparation. 


\section{EBID Lithography}

The nanofabrication sequence is summarized in Figure 1, top. First, an SOI layer is reduced to a thin silicon film by plasma etching and the EBID lithography is carried out on this area. Then the formed metal pattern is transferred to the silicon layer by second plasma etching. Finally, the metal mask is chemically removed and resulting nanostructures are passivated with silicon dioxide. Below on the same figure an optical image of the SOI substrate is shown after the first plasma etching step. It is common that the plasma etching rate is not uniform across the chip with a higher rate at the sample edge. This non-uniformity leads to the formation of a very thin transition layer in between the overetched part and remaining silicon in the middle of the sample. The location of this region can be easily identified in an optical microscope under white light illumination due to different color of films of different thickness. For example, a pale blue area in Figure 1 corresponds to a silicon layer less than $10 \mathrm{~nm}$ thick. All metal depositions were performed on these areas assuring the appropriate size of nanostructures in the vertical dimension. In general, however, uniform ultrathin SOI wafers (with $\sim 20 \mathrm{~nm}$ SOI layer) can be also used for this purpose after controllable short oxidation and oxide removal.

In Figure 2 nanodeposit shape evolution with increasing beam dwell time is presented. For this purpose metal nanodots were deposited on a silicon membrane thin edge for TEM observation (Fig. 2, top). Altogether eleven nanodots with one second increment step were 
produced, starting from the two seconds dwell time nanostructure. It is seen that the base diameter grows linearly with increasing dose (Fig. 2, bottom). This parameter is primarily responsible for the resulting semiconductor nanostructure size since it limits the etching mask. Results for two different deposition runs are very similar, suggesting good reproducibility of such deposition routine. It is also seen that under present deposition conditions metal nanostructures formed with less than 8 seconds dwell time are already in the sub-10 nm range.

The deposition is believed to occur when the electron beam decomposes adsorbed gas molecules on the sample surface forming carbon-based volatile compounds and leaving metal deposits behind. The surface diffusion is a main supply source of precursor gas molecules to the deposition site. At the same time carbonaceous compounds on the sample surface can also contribute to the deposition process, forming polymerized carbon inclusions in the nanodeposits.

\section{Results and discussion}

In Figure 3 cross-sectional bright field TEM images of silicon nanodots (lattice fringes visible) are shown after the second plasma etching with metal dots (dark) still remaining on top. These nanostructures reside on the buried oxide of the SOI wafer and are surrounded by a 
protective carbon film deposited before the FIB milling. Metal dots prepared by EBID have a Gaussian-shape as would be expected from the Monte-Carlo simulations [21]. Previously the role of carbon contaminations on the nanodeposit structure and composition was investigated in detail [22]. It was shown how carbon inclusions affecting nanodeposit size can be reduced by sample pre-heating. Here we present results of the EBID deposition on as-prepared and pre-heated substrates from a lithographical perspective. In Figure 3, left, just below the metal dot one can distinguish a carbon "basement", formed during the deposition. This effective broadening of the mask leads to a remarkable increase in the silicon nanostructure size after the etching. On the other hand there is no such an effect for a pre-heated sample (Figure 3, right), attributed to the suppressed carbonaceous compound diffusion to the deposition site. Statistical data related to this phenomenon are shown in Figure 4. Three different runs made on as-prepared samples (open symbols) yield large silicon nanostructures, on average two times the mask size. Pre-heating, however, has a strong effect and two different runs carried out on a treated substrate (dark symbols) resulted in smaller Si nanostructures. It is important to note that their size also depends on the etching time. This fact indicates that the silicon part is well exposed to the fluorine ions of the plasma unlike in the case of the untreated sample. After a longer etching the silicon-to-mask diameter ratio can be even pushed below unity as seen from the results of the 35 seconds etching run. The fact that carbon can also act as an etching mask 
suggests that it can be used instead of metalorganic precursor gas. However the amount of already existing carbonaceous compounds on the sample surface depends on its history. Hence the reproducibility of such nanofabrication would be worse than in the case of controlled gas supply of the metalorganic precursor used here.

Altogether it shows that surface contaminations are an important part of the deposition process and special care is needed to avoid their undesired involvement. Thus, in practice, silicon nanostructure size can be controlled by both the metal mask size (base diameter) and the plasma etching time for a pre-heated sample. By tuning these two essential parameters a regime was found where sub-10 $\mathrm{nm}$ silicon nanostructures can be reproducibly fabricated. The statistical nature of the deposition and etching processes yields some deviation of the final silicon nanostructure size from its average value shown by straight lines in Figure 4 .

In Figure 5 typical TEM images of silicon nanodots at the final stages of the processing are shown. After a short dip in the heated "aqua regia" solution only semiconductor nanodots remain while the metal part is completely removed (Figure 5, left). A subsequent rapid thermal oxidation for 10 seconds at $900^{\circ} \mathrm{C}$ forms a thin dioxide layer around nanodots (Figure 5, right). This surface passivation is vital for functionalization of silicon nanostructures whether for optical or electrical applications. Non-radiative recombination through surface defect states can 
degrade light emission properties, while an undesired charge trapping can be detrimental to the conductivity performance. We note that, although thermal oxidation is a well-known method for size reduction of silicon nanostructures [13], here it is only used for surface passivation since the initial nanostructure size is already below $10 \mathrm{~nm}$.

We also show that silicon nanowires can be fabricated in a similar way as nanodots. A metal dot formed with 2 seconds e-beam dwell time is on average of a $5 \mathrm{~nm}$ diameter at the basement (cf. Fig. 2). A number of such metal dots can be placed in a sequence to form a nanowire template as shown in Figure 6, inset. For the fabrication of a continuous wire the spacing between the dots was chosen to be $3.5 \mathrm{~nm}$. As a result a sub-10 nm wide metal nanowire of a desired length can be produced. A relatively long deposition time requires a sample drift compensation routine to be employed for the deposition of a straight wire. Deposition time, however, can be significantly reduced in the tilted angle deposition geometry, where the beam hits the surface at an oblique angle. In this case a larger fraction of the beam energy is utilized for the precursor gas molecule decomposition [23]. In Figure 6 a cross-section TEM image of a $~ 100 \mathrm{~nm}$ long silicon nanowire fragment is shown. The length of an observable nanowire in cross-sectional TEM is limited by the lamella thickness transparent for the electron beam. The grey region around silicon nanowire in Figure 6 corresponds to silicon dioxide, as confirmed by EELS (not shown), 
although no thermal oxidation was carried out in this case. It is known, however, that the "aqua regia" solution itself can oxidize silicon at a slow rate and a longer dip used here for a large mask removal resulted in the observed oxide layer.

Finally we note that any pattern combining nanodots and nanowires should be possible to realize with this method. For example closely spaced dots and nanowire-nanodot hybrid structures can be fabricated for e.g. electric carrier transport studies.

The novel nanofabrication method described here can serve as an ultimate tool for the manufacturing of nanostructures with small dimensions. By using electron beam induced deposition metal nanostructures were formed on a thin SOI substrate. Their size and purity was controlled by sample pre-treatment and beam dose. The pattern was successfully transferred to the silicon layer by dry etching and resulting nanostructures were visualized in a transmission electron microscope. Thus it was shown how sub-10 nm monocrystalline silicon nanodots and nanowires with passivated surface can be fabricated in a controllable manner. Semiconductor and metal nanostructures can be intrinsically combined by this method, making a large diversity of specific designs and prototype devices possible to produce.

\section{Acknowledgements}


This research was supported by Ministry of Education, Science, Sports and Culture (MEXT)

through a Grant-in-Aid for Scientific Research(C) 21560036 and also partially supported by the "Nanotechnology Network Project" of MEXT. 


\section{Figure Captions}

Figure 1 (top) Nanofabrication process flow, from left to right: SOI layer thinning by plasma etching; metal patterning by EBID; SOI layer etching with a metal mask and subsequent mask removal; oxidation of nanostructures for surface passivation. (bottom) An optical microscope image of an SOI chip after first plasma etching with a silicon layer thickness gradient. The silicon layer thickness is increasing from right top to left bottom of the image, where the pale blue color area corresponds to a few nanometers Si film. EBID patterning was usually performed on this region.

Figure 2 (top) TEM image of as-deposited metal nanodots on a silicon membrane thin edge made with increasing beam dose, from left to right. (bottom) Metal dot size as a function of the beam dwell time for two different deposition runs (circles and squares). Base diameter defines mask size in the subsequent etching process.

Figure 3 Cross-sectional TEM images of Si nanodots with a W mask remaining on top after second plasma etching. The same size of the metal mask results in different size of silicon nanostructures depending on the surface pre-treatment: (left) for an as-prepared sample carbon "basement" formed during the deposition acts as a mask itself, yielding a large Si nanodot; 
(right) for a pre-heated sample silicon nanodots similar in size to the mask can be fabricated.

Figure 4 Fabricated semiconductor nanostructure size as a function of the metal mask size for samples with different pre-treatment. Open triangles, squares and circles represent three different runs under identical conditions on an as-prepared substrate. On average, the silicon nanostructure size is more than double that the size of the mask, regardless of the etching time. Filled triangles and circles correspond to two different runs with varying etching time (30 and 35 seconds) on a pre-heated substrate. In this case the silicon nanodot size is similar to that of the mask and, furthermore, dependent to the etching time. Dotted line marks the border of the quantum confinement size range for silicon (less than $10 \mathrm{~nm}$ ).

Figure 5 Cross-sectional TEM images of silicon nanodots at the final stages of the nanofabrication process: (left) after metal mask removal, and (right) after rapid thermal oxidation.

Figure 6 Cross-sectional TEM images of silicon nanowires fabricated by EBID lithography and plasma etching; (inset) top-view SEM image of the initial metal mask. 


\section{References}

[1] Hodes G 2007 Adv. Mat. 19639

[2] Ieong M, Doris B, Kedzierski J, Rim K, Yang M 2004 Science 3062057

[3] Sychugov I, Juhasz R, Valenta J and Linnros J 2005 Phys. Rev. Lett. 94087405

[4] Nakajima Y, Takahashi Y, Horiguchi S, Iwadate K, Namatsu H, Kurihara K and Tabe M 1995 Jpn. J. Appl. Phys. 34, 1309.

[5] Tiwari S, Rana F, Hanafi H, Hartstein A, Crabbe EF and Chan K 1995 Appl. Phys. Lett. 68 1377; Ohba R, Sugiyama N, Uchida K, Koga J and Toriumi A 2002 IEEE Trans. Electron Devices 491392.

[6] Li Q, Zhu X, Xiong HD, Koo S-M, Ioannou DE, Kopanski JJ, Suehle JS and Richter CA 2007 Nanotechnology 18235204

[7] Elfstrom N, Juhasz R, Sychugov I, Engfeldt T, Karlstrom AE and Linnros J 2007 Nano Lett. 72608

[8] Conibeer G, Green M, Corkish R, Cho Y, Cho E-C, Jiang C-W, Fangsuwannarak T, Pink E, Huang Y, Puzzer T, Trupke T, Richards B, Shalav A and Lin K-L 2006 Thin Solid Films 511654

[9] Erogbogbo F, Yong K-T, Roy I, Xu G, Prasad PN and Swihart MT 2008 ACS Nano 2873.

[10] Boukai A, Bunimovich Y, Tahir-Kheli J, Yu JK, Goddard III WA, Heath JR 2008 Nature 451168. 
[11] Hallstedt J, Hellstrom P-E, Zhang Z, Malm BG, Edholm J, Lu J, Zhang SL, Radamson HH and Ostling M 2006 Microelectron. Eng. 83 434; Chen S, Bomer JG, van der Wiel WG, Carlen ET and van der Berg A 2009 ACS Nano 33485.

[12] Namatsu H, Watanabe Y, Yamazaki K, Yamaguchi T, Nagase M, Ono Y, Fujiwara A, Horiguchi S 2003 J. Vac. Sci. Technol. B 211

[13] Juhasz R, Elfstrom N and Linnros J 2005 Nano Lett. 5275.

[14] Sheu JT, Kuo JM, You KS, Chen CC and Chang KM 2004 Microelectron. Eng. 73594.

[15] Koops HWP, Weiel R, Kern DP and Baum TH 1988 J. Vac. Sci. Technol. B 6477

[16] van Dorp WF and Hagen CW 2008 J. Appl. Phys. 104 081301; Utke I, Hoffmann P and Melngailis J 2008 J. Vac. Sci. Tech. B 26, 1197.

[17] Mitsuishi K, Shimojo M, Tanaka M and Furuya K 2006 Nucl. Instrum. Methods Phys. Res. Sect. B-Beam Interact. Mater. Atoms 242244

[18] Guan Y, Fowlkes JD, Retterer ST, Simpson ML and Rack PD 2008 Nanotechnology 19 505302

[19] Broers AN, Molzen WW, Cuomo JJ and Wittels ND 1976 Appl. Phys. Lett. 29596.

[20] Wang J, Ma X.-C., Lu L, Jin A.-Z., Gu C.-Z., Xie X.C., Jia J.-F., Chen X., Xue Q-K. 2008 Appl. Phys. Lett. 92233119.

[21] Liu ZQ, Mitsuishi K and Furuya K 2006 Nanotechnology 173832 
[22] Sychugov I, Nakayama Y and Mitsuishi K 2009 J. Phys Chem. C 11321516

[23] Sychugov I, Nakayama Y and Mitsuishi K 2010 Nanotechnology 21025303 

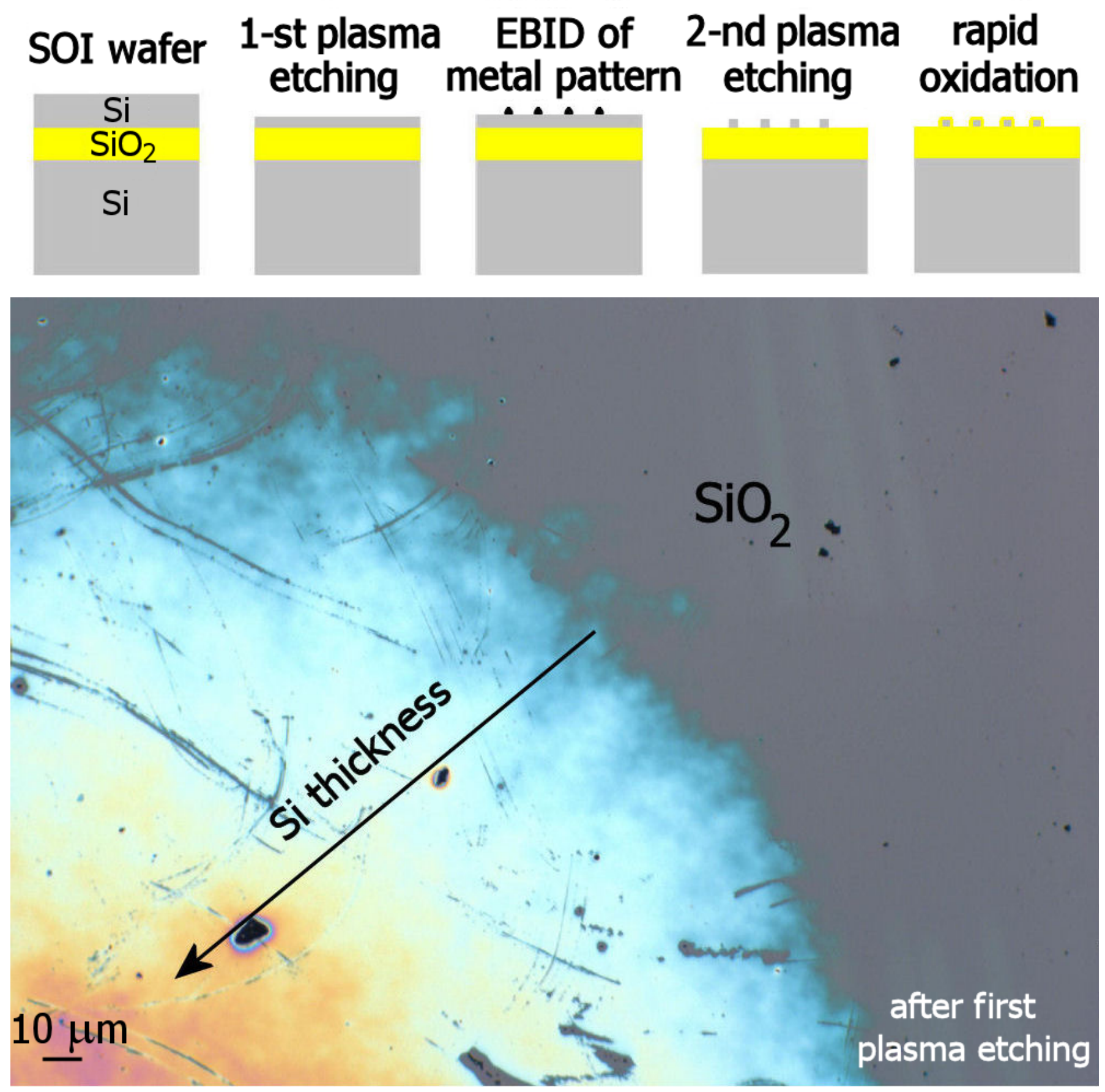

Figure 1 (Fig1.tif) 


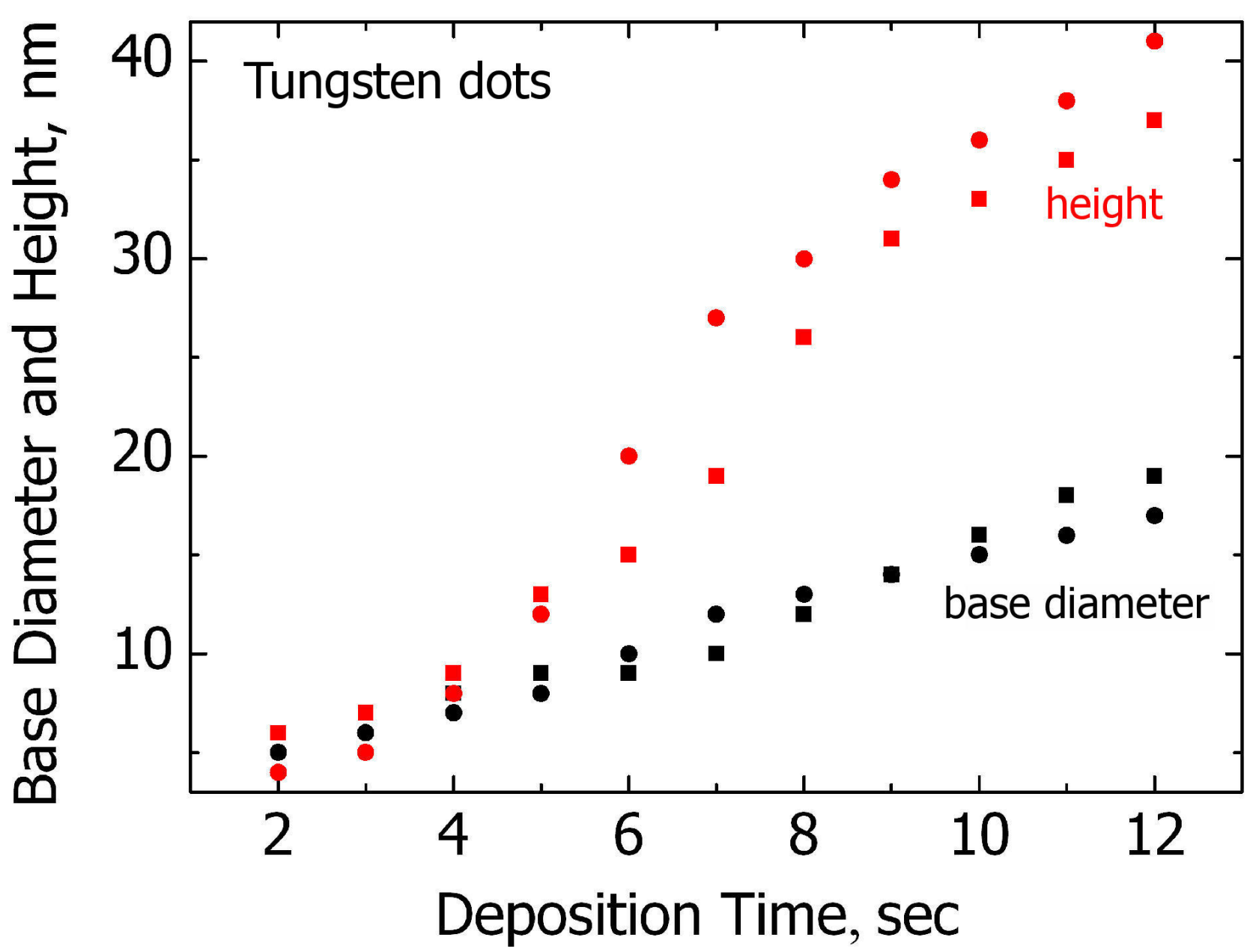

Figure 2 (Fig2.tif) 


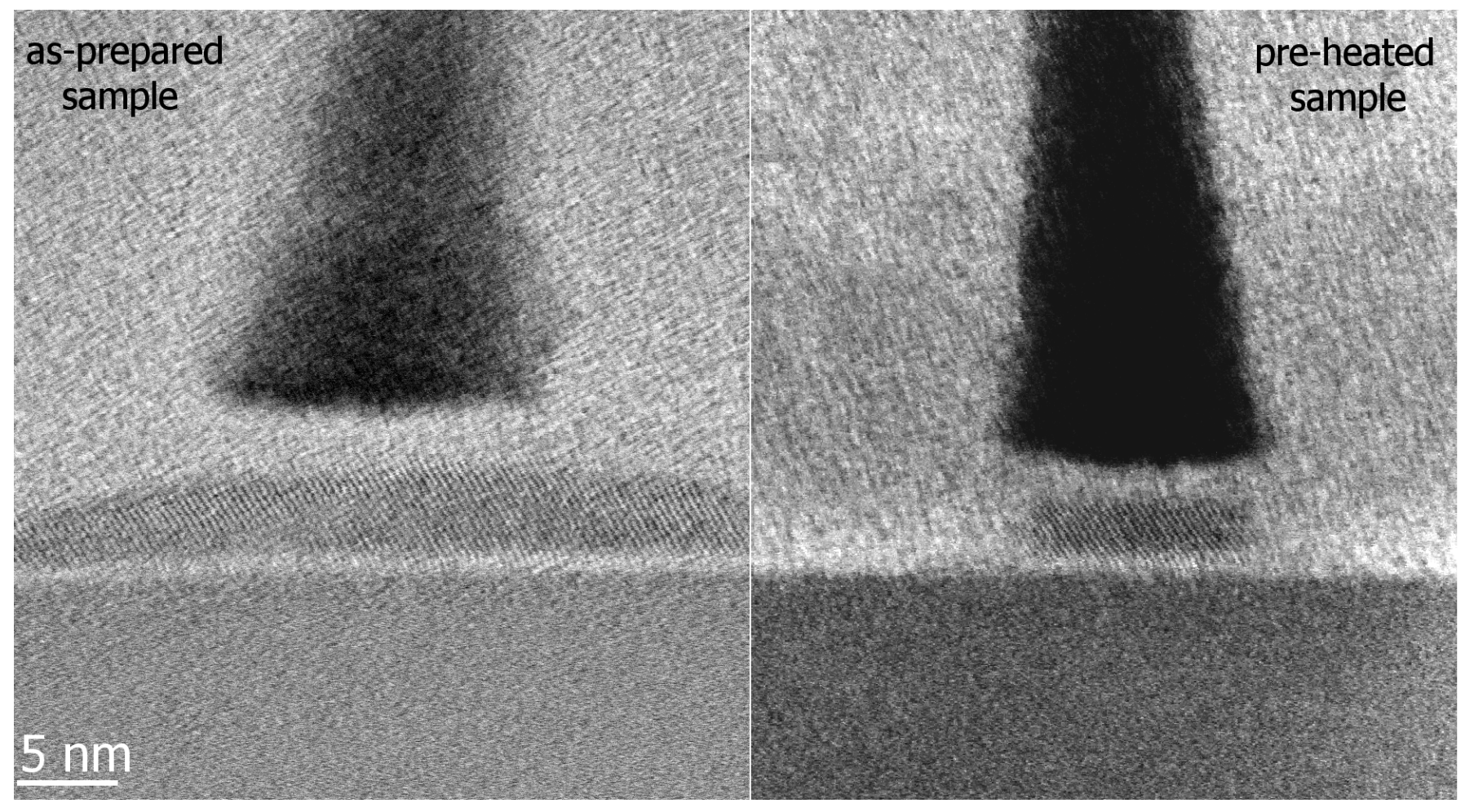

Figure 3 (Fig3.tif) 


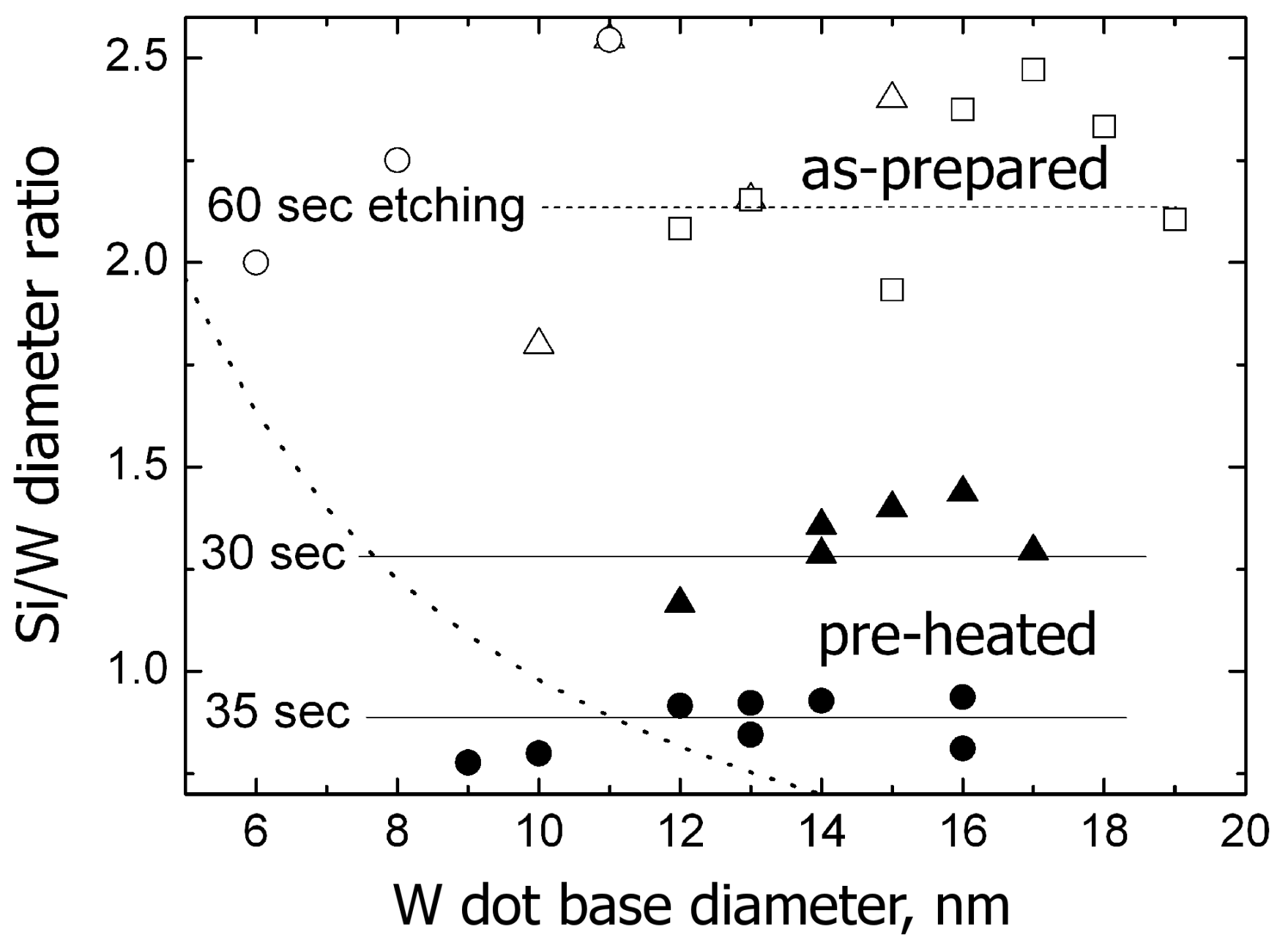

Figure 4 (Fig4.tif) 


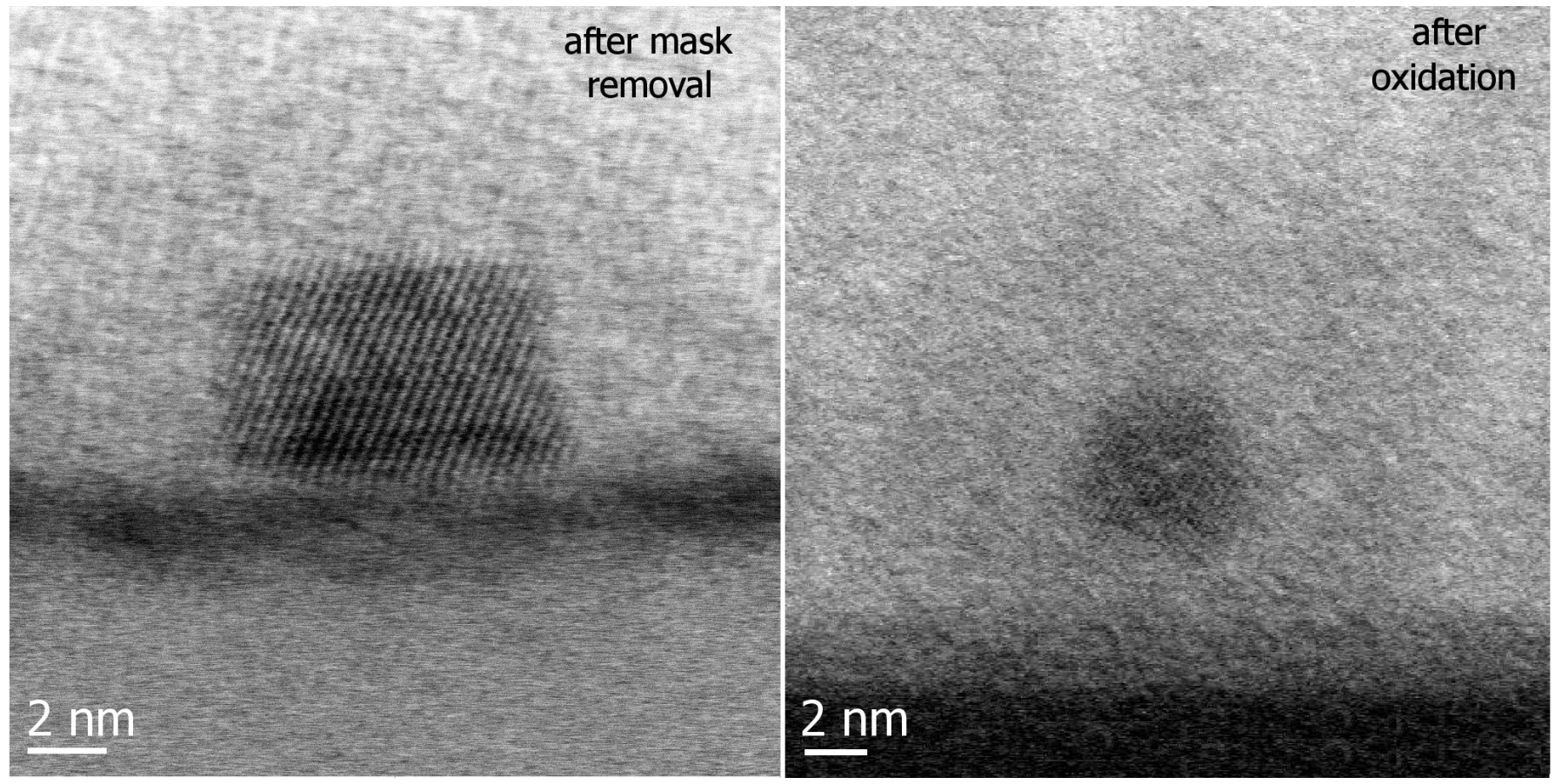

Figure 5 (Fig5.tif) 


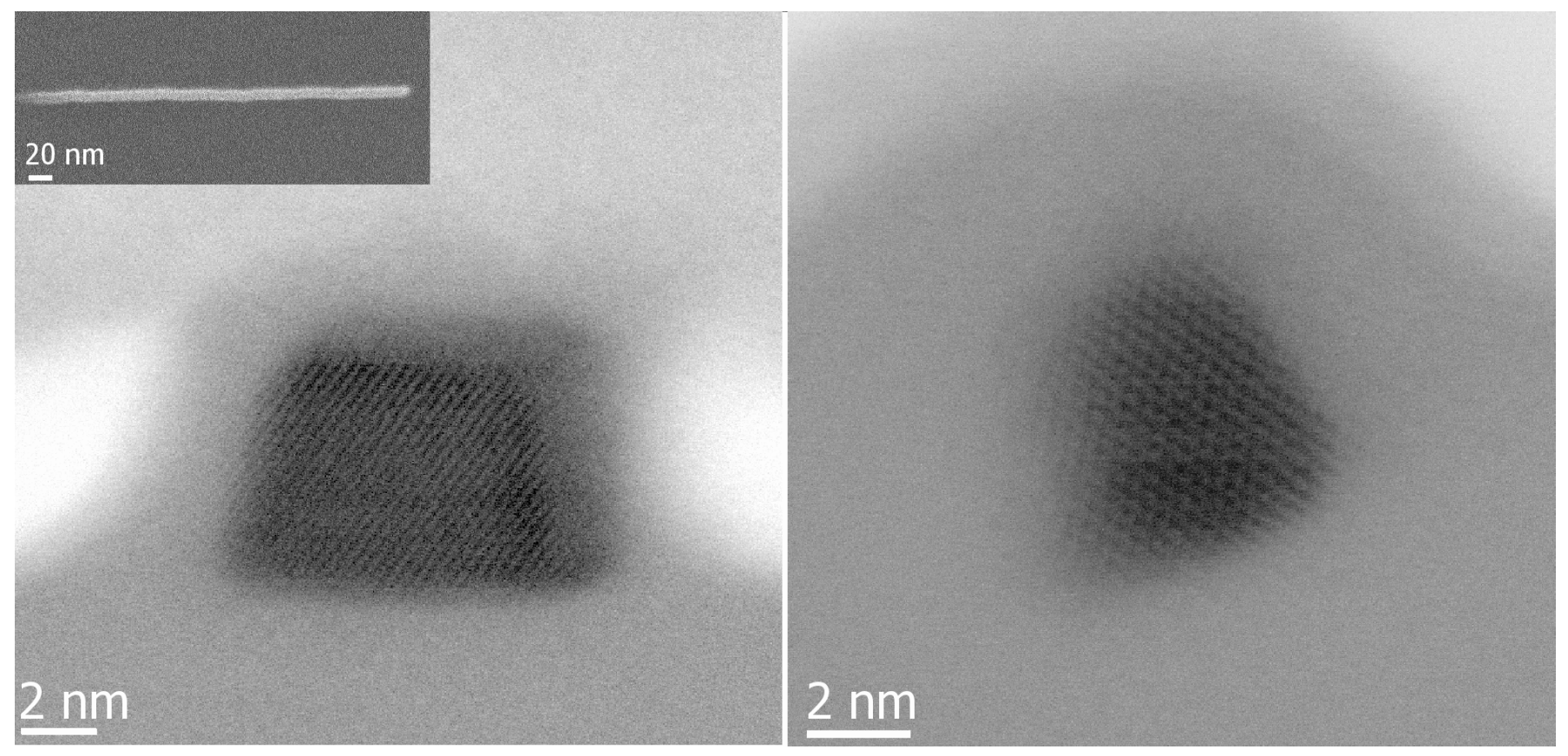

Figure 6 (Fig6.tif) 